\title{
Criteria for confounders in epidemiological studies
}

\author{
Zhi Geng, \\ Peking University, Beijing, People's Republic of China \\ Jianhua Guo \\ Northeast Normal University, Changchun, People's Republic of China \\ and Wing-Kam Fung \\ University of Hong Kong, People's Republic of China
}

[Received February 2000. Final revision July 2001]

\begin{abstract}
Summary. The paper addresses a formal definition of a confounder based on the qualitative definition that is commonly used in standard epidemiology text-books. To derive the criterion for a factor to be a confounder given by Miettinen and Cook and to clarify inconsistency between various criteria for a confounder, we introduce the concepts of an irrelevant factor, an occasional confounder and a uniformly irrelevant factor. We discuss criteria for checking these and show that Miettinen and Cook's criterion can also be applied to occasional confounders. Moreover, we consider situations with multiple potential confounders, and we obtain two necessary conditions that are satisfied by each confounder set. None of the definitions and results presented in this paper require the ignorability and sufficient control confounding assumptions which are commonly employed in observational and epidemiological studies.
\end{abstract}

Keywords: Causal effect; Comparability; Confounding; Counterfactual model; Epidemiological methods

\section{Introduction}

The concepts of confounders and confounding are of great importance in epidemiology (Kleinbaum et al., 1982; Rothman, 1986; Greenland, Robins and Pearl, 1999). In the presence of confounding, the effect of exposure on the rate of a disease cannot be assessed correctly. Criteria for assessing a confounder and confounding still appear to be inconsistent in the epidemiological literature (Boivin and Wacholder, 1985; Grayson, 1987; Greenland et al., 1989; Weinberg, 1993; Greenland, Pearl and Robins, 1999; Greenland, Robins and Pearl, 1999). According to Greenland and Robins (1986), there are basically two main approaches for assessing confounding and a confounder. One approach, called 'collapsibility based', considers confounding as arising from differences between stratified measures of association and the corresponding crude measure. The collapsibility-based approach has a criterion for a confounder based on the collapsibility of a particular parameter of association (Breslow and Day, 1980; Kleinbaum et al., 1982). This approach depends on both the parameter of interest and the categorization of the factor to be collapsed over. For the use of a collapsibility criterion in the analysis of contingency tables and statistical modelling, see for example

\footnotetext{
Address for correspondence: Zhi Geng, Department of Probability and Statistics, Peking University, Beijing 100871, People's Republic of China.

E-mail: zgeng@math.pku.edu.cn
} 
Whittemore (1978), Ducharme and LePage (1986), Geng (1992) and Guo and Geng (1995). The other approach, called 'comparability based', considers confounding as arising from the exposed and unexposed populations which are not comparable. We say that the unexposed population is comparable with the exposed population if the proportion of diseased individuals in the unexposed population equals the hypothetical proportion of diseased individuals in the exposed population without exposure. The comparability-based approach determines a factor to be a confounder if adjusting for it reduces confounding (Greenland and Robins, 1986; Greenland, Robins and Pearl, 1999). The comparability-based approach has the following criterion for a confounder which was obtained inductively by Miettinen and Cook (1981) (see also Greenland and Robins (1986)):

(a) it must be predictive of risk in the unexposed population and

(b) it must have different distributions between the exposed and unexposed populations.

Greenland and Robins (1986) illustrated that this criterion is not sufficient for a confounder.

Greenland, Pearl and Robins (1999) described a causal diagram approach for detecting multiple confounders under the assumption that the causal diagram is completely constructed on the basis of knowledge beyond the empirical data and that it includes a sufficient set of potential confounders. The causal diagram approach presented by Spirtes et al. (1993) and Pearl (1995) was originally intended for assessing a causal effect of an external intervention on the whole population, including both exposed and unexposed individuals. As many researchers have mentioned (Miettinen and Cook, 1981; Greenland and Robins, 1986; Wickramaratne and Holford, 1987; Holland, 1989), epidemiological studies are more concerned with causal effects of exposure in the exposed population. Thus, in such an epidemiological study, we construct a control group which would be comparable with the exposed population if the exposure were absent in that population.

In this paper, we give a formal definition of a confounder according to the standard epidemiological definition of a confounder presented by Miettinen and Cook (1981), Kleinbaum et al. (1982), Greenland and Robins (1986) and Greenland, Robins and Pearl (1999). This definition does not require the assumption that a known set of potential confounders is sufficient for the control of confounding. We first focus on the situations with a single potential confounder and show that Miettinen and Cook's criterion for a confounder does not require any untestable assumptions. Then we illustrate inconsistency between Miettinen and Cook's criterion and the criterion based on the collapsibility of differences in risk or relative risks. To clear up this inconsistency, we introduce the concepts of an occasional confounder and uniformly irrelevant factor. Finally we extend these results to situations with multiple potential confounders.

In Section 2, we formalize the definitions of a confounder and an irrelevant factor on the basis of the counterfactual model presented by Rubin (1974) and Holland (1986). Section 3 gives the necessary and sufficient condition for a uniformly irrelevant factor and shows that Miettinen and Cook's criterion is a necessary condition for an occasional confounder. In Section 4 we discuss how Miettinen and Cook's criterion can be applied to situations with multiple potential confounders. A few concluding remarks are given in Section 5.

\section{Confounder and irrelevant factor}

We follow the notation of Holland (1989). Let $E$ be an exposure with values $e$ and $\bar{e}$ representing its presence or absence respectively, and let $D_{e}$ and $D_{\bar{e}}$ be the corresponding responses, which take values 1 or 0 denoting the presence or absence of a disease. 
Epidemiological studies focus on the effects of exposure on the rate of a disease in the exposed population. Let $P\left(D_{e}=1 \mid E=e\right)$ and $P\left(D_{\bar{e}}=1 \mid E=\bar{e}\right)$ be the proportions of diseased individuals in the exposed population and the unexposed or control population, and let $P\left(D_{\bar{e}}=1 \mid E=e\right)$ be the hypothetical proportion of individuals in the exposed population who would have developed the disease even if they had not been exposed. Since $P\left(D_{\bar{e}}=1 \mid E=e\right)$ is a hypothetical proportion, the model is a counterfactual model (Rubin, 1974; Holland, 1986).

As mentioned in Greenland and Robins (1986), confounding arises from inherent differences in risk between the exposed and unexposed populations. The term inherent differences' here means differences that would exist even if exposure were entirely absent from both populations. Confounding bias $B$ is defined as the difference between the hypothetical proportion of diseased individuals in the exposed population without exposure and the proportion of diseased individuals in the unexposed population (Wickramaratne and Holford, 1987; Holland, 1989), i.e.

$$
B=P\left(D_{\bar{e}}=1 \mid E=e\right)-P\left(D_{\bar{e}}=1 \mid E=\bar{e}\right) .
$$

If $B=0$, then the exposed and unexposed populations are comparable and there is no confounding (Wickramaratne and Holford, 1987). However, we cannot guarantee $B=0$ for an observational study. In this case, we try to adjust for some factors to estimate the hypothetical proportion.

Let $C$ be a factor with possible values $1, \ldots, K$; assume that $C$ is not an intervening variable in a causal pathway from exposure to disease, i.e. $C$ is a covariate. Let $\Delta=\{1, \ldots, K\}$, and let the subpopulations be defined by $k \in \Delta$. Assume that $P(E, C)>0$ for all possible values of $E$ and $C$. Define $P\left(D_{\bar{e}}=1 \mid E=\bar{e}, C=k\right)$ to be the proportion of diseased individuals in the unexposed subpopulation of $C=k$, and $P\left(D_{\bar{e}}=1 \mid E=e, C=k\right)$ the hypothetical proportion of diseased individuals in the exposed subpopulation $C=k$ if exposure were entirely absent. Confounding bias in the subpopulation $C=k$ is defined as

$$
B_{k}=P\left(D_{\bar{e}}=1 \mid E=e, C=k\right)-P\left(D_{\bar{e}}=1 \mid E=\bar{e}, C=k\right) .
$$

When there is no confounding in any subpopulation, then $B_{k}=0$ for all $k$, and it means that each unexposed subpopulation is comparable with the corresponding exposed subpopulation. This situation is called subpopulation comparable, also called sufficient control confounding in Greenland, Robins and Pearl (1999). Subpopulation comparability is equivalent to the case when $D_{\bar{e}}$ is independent of $E$ given $C$, denoted by $D_{\bar{e}} \Perp E \mid C$ in the notation of Dawid (1979). It is related to the weak and strong ignorability assumptions defined by Rosenbaum and Rubin (1983). Weak ignorability is defined as $D_{\bar{e}} \Perp E \mid C$ and $D_{e} \Perp E \mid C$, and strong ignorability is defined as $\left(D_{\bar{e}}, D_{e}\right) \Perp E \mid C$. Thus strong ignorability implies weak ignorability, which in turn implies subpopulation comparability. None of these can be verified from empirical data (Rubin, 1974; Greenland, 1989; Holland, 1986, 1989).

\subsection{Definitions}

The common definition of a confounder is that it is a risk factor for the disease, control of which can reduce bias for estimating causal effects (see Miettinen and Cook (1981), Kleinbaum et al. (1982), page 244, and Greenland and Robins (1986)). Here 'control' means adjustment at the analysis stage or stratification at the design stage of a study.

We now formalize the common definition of confounders. According to the common standardization in epidemiology (Miettinen, 1972; Kleinbaum et al., 1982; Rothman, 1986), the standardized proportion $P_{\Delta}\left(D_{\bar{e}}=1 \mid E=\bar{e}\right)$ obtained by adjusting the distribution of $C$ in the unexposed population to that in the exposed population is 


$$
P_{\Delta}\left(D_{\bar{e}}=1 \mid E=\bar{e}\right)=\sum_{k=1}^{K} P\left(D_{\bar{e}}=1 \mid E=\bar{e}, C=k\right) P(C=k \mid E=e) .
$$

Definition 1. A covariate $C$ is a confounder if

$$
\left|P\left(D_{\bar{e}}=1 \mid E=e\right)-P_{\Delta}\left(D_{\bar{e}}=1 \mid E=\bar{e}\right)\right|<|B| .
$$

This definition states that the standardized proportion $P_{\Delta}\left(D_{\bar{e}}=1 \mid E=\bar{e}\right)$ obtained by adjusting for the confounder $C$ is closer to the hypothetical proportion $P\left(D_{\bar{e}}=1 \mid E=e\right)$ than is the crude proportion $P\left(D_{\bar{e}}=1 \mid E=\bar{e}\right)$. It does not need the subpopulation comparable assumption. Like Miettinen and Cook's (1981) criterion, this definition considers a single potential confounder or a composite confounder consisting of several potential confounders. Situations with multiple potential confounders are discussed in Section 4. As a referee has noted, under this definition it is possible that $C_{1}$ is a confounder, $C_{2}$ is a confounder but $\left\{C_{1}\right.$, $\left.C_{2}\right\}$ is not a confounder. To avoid this counter-intuitive property, we present the concept of an occasional confounder in the next section. Note that confounding bias may not be completely eliminated by controlling a confounder, whereas there may be an unbiased estimator without controlling for the confounder. It requires an untestable assumption (such as a causal diagram or a sufficient set of potential confounders) to determinate an unbiased estimator.

To study the concept of a confounder analytically, the idea of an irrelevant factor is also required.

Definition 2. A covariate $C$ is an irrelevant factor if

$$
P_{\Delta}\left(D_{\bar{e}}=1 \mid E=\bar{e}\right)=P\left(D_{\bar{e}}=1 \mid E=\bar{e}\right)
$$

Since the estimate of the hypothetical proportion remains unchanged after adjusting for an irrelevant factor, we do not need to adjust for it to reduce confounding. A factor may be neither a confounder nor an irrelevant factor, and such factors should not be controlled for since confounding will be increased by controlling for it; an example was given in Greenland and Robins (1986). Robins and Morgenstern (1987), page 873, considered an alternative approach which defines a factor to be a confounder if it is not an irrelevant factor.

\subsection{Criteria}

Let $C \Perp E$ denote independence of $C$ and $E$, and $D_{\bar{e}} \Perp C \mid E=\bar{e}$ denote conditional independence of $D_{\bar{e}}$ and $C$ given $E=\bar{e}$.

Proposition 1. If $C$ is a confounder, then

(a) $C \not \perp E$ and

(b) $D_{\bar{e}} \not \Perp C \mid E=\bar{e}$.

Proof. Suppose that $C \Perp E$ or $D_{\bar{e}} \Perp C \mid E=\bar{e}$ holds. We can obtain immediately that $C$ is an irrelevant factor and thus not a confounder.

Proposition 1 verifies the criterion for a confounder obtained inductively by Miettinen and Cook (1981), with the following qualitative meanings.

(a) $D_{\bar{e}} \not \perp C \mid E=\bar{e}: C$ is predictive of risk in the unexposed population.

(b) $C \not \Perp E$ : $C$ has different distributions in the exposed and unexposed populations.

Wickramaratne and Holford (1987) stated that the conditions $C \Perp E$ or $D_{\bar{e}} \Perp C \mid E=\bar{e}$, which are the converse of Miettinen and Cook's criterion for a confounder, are sufficient for 
non-confounding under the subpopulation comparable assumption. Robins (1989) showed, without this assumption, that the conditions $C \Perp E$ or $D_{\bar{e}} \Perp C \mid E=\bar{e}$ are sufficient for the equality of the standardized risk difference and the crude risk difference.

\section{Occasional confounder and uniformly irrelevant factor}

The above definitions of a confounder and an irrelevant factor depend on the choice of categorization (i.e. level or scale) for the factor under consideration. For example, age may be a confounder or an irrelevant factor when it is categorized by every 10 years of age, but it may not be a confounder when categorized by every 20 years. Such a phenomenon is illustrated in the example given in Section 3.1. When there is confounding in subpopulations induced by a potential confounder, we can see from this example that non-collapsibility of risk differences is neither equivalent to Miettinen and Cook's (1981) criterion nor a necessary condition of a confounder, whereas Miettinen and Cook's criterion is still necessary not only for a confounder as shown in Section 2.2 but also for an occasional confounder, to be shown in this section.

\subsection{An example}

Let a factor $C$ indicate groups categorized by every 10 years of age, and its values 1, 2, 3 and 4 denote the original age groups 20-29, 30-39, 40-49 and 50-59 years respectively. Using the individual effect model presented by Greenland and Robins (1986), we can classify all individuals into the following four types:

(a) type 1 , no effect (individual 'doomed'), $D_{e}=D_{\bar{e}}=1$;

(b) type 2, exposure causative (individual susceptible), $D_{e}=1$ and $D_{\bar{e}}=0$;

(c) type 3, exposure preventive (individual susceptible), $D_{e}=0$ and $D_{\bar{e}}=1$;

(d) type 4, no effect (individual immune to disease), $D_{e}=D_{\bar{e}}=0$.

Suppose that there is no exposure effect, i.e. there are only individuals of types 1 and 4 , and that the joint distribution of disease, exposure and a factor $C$ is given in Table 1. Since $B=156 / 300-174 / 300=-0.06$, there is confounding. Since $P\left(D_{\bar{e}}=1 \mid E=\bar{e}\right)=0.58=$ $P_{\Delta}\left(D_{\bar{e}}=1 \mid E=\bar{e}\right), C$ is an irrelevant factor and bias cannot be reduced by adjusting the distribution of $C$.

When the individuals are regrouped by every 20 years, we obtain a coarse subpopulation as given in Table 2. For the regrouping denoted by $p=\{\{1,2\},\{3,4\}\}$, the standardized $P_{p}\left(D_{\bar{e}}=1 \mid E=\bar{e}\right)$ is

$$
P_{p}\left(D_{\bar{e}}=1 \mid E=\bar{e}\right)=\frac{34}{100} \frac{150}{300}+\frac{140}{200} \frac{150}{300}=0.52 .
$$

In this case of no exposure effect, we also find that $P\left(D_{\bar{e}}=1 \mid E=e\right)=P\left(D_{e}=1 \mid E=e\right)=$ 0.52 . Since $\left|P\left(D_{\bar{e}}=1 \mid E=e\right)-P_{p}\left(D_{\bar{e}}=1 \mid E=\bar{e}\right)\right|=0$ is less than $|B|=0.06$, we find that $C$ is a confounder with respect to the categorization of every 20 years. Confounding can be corrected since $P\left(D_{\bar{e}}=1 \mid E=e\right)=P_{p}\left(D_{\bar{e}}=1 \mid E=\bar{e}\right)$.

It can be seen from this example that there are some situations in which confounding can be reduced by recategorizing a potential confounder, although we cannot recognize these situations from observed data. However, this example by no means suggests that we should try to merge the levels of a factor to correct confounding. It should be noted that the risk differences in Table 1 are collapsible over groups of 10 years but Miettinen and Cook's (1981) criterion is satisfied. Thus these collapsibility- and comparability-based approaches 
Table 1. Distribution with age group $C$ being a non-confounder

\begin{tabular}{|c|c|c|c|c|c|c|c|c|c|c|}
\hline \multirow[t]{3}{*}{ Type } & \multicolumn{8}{|c|}{ Distribution for the following values of $C$ : } & \multicolumn{2}{|c|}{ Total $(C \in \Delta \dagger)$} \\
\hline & \multicolumn{2}{|c|}{$C=1$} & \multicolumn{2}{|c|}{$C=2$} & \multicolumn{2}{|c|}{$C=3$} & \multicolumn{2}{|c|}{$C=4$} & \multirow[t]{2}{*}{$E=e$} & \multirow[t]{2}{*}{$E=\bar{e}$} \\
\hline & $E=e$ & $E=\bar{e}$ & $E=e$ & $E=\bar{e}$ & $E=e$ & $E=\bar{e}$ & $E=e$ & $E=\bar{e}$ & & \\
\hline 1 ('doomed') & 5 & 8 & 46 & 26 & 82 & 88 & 23 & 52 & 156 & 174 \\
\hline 4 ('immune') & 45 & 42 & 54 & 24 & 18 & 12 & 27 & 48 & 144 & 126 \\
\hline Total & 50 & 50 & 100 & 50 & 100 & 100 & 50 & 100 & 300 & 300 \\
\hline
\end{tabular}

$\dagger \Delta=\{1, \ldots, 4\}$.

Table 2. Distribution with $C$ being a confounder with respect to partition $p=$ $\{\{1,2\},\{3,4\}\}$

\begin{tabular}{|lcccc|}
\hline \multirow{2}{*}{ Type } & \multicolumn{3}{c|}{ Distribution for the following values of $C$ : } \\
\cline { 2 - 5 } & \multicolumn{2}{c|}{$C \in\{1,2\}$} & & $C \in\{3,4\}$ \\
\cline { 2 - 5 } & $E=e$ & $E=\bar{e}$ & $E=e$ & $E=\bar{e}$ \\
\cline { 2 - 5 } & 51 & 34 & 105 & 140 \\
4 ('doomed') & 99 & 66 & 45 & 60 \\
Total & 150 & 100 & 150 & 200 \\
\hline
\end{tabular}

are inconsistent for detecting whether $C$ is a confounder. We can eliminate this inconsistency by introducing the concepts of an occasional confounder and uniformly irrelevant factor.

This example is mainly used to illustrate the fact that a factor may be detected as a confounder in one study but not in another owing to using measurements on different scales. Even in the same study, different people may reach different conclusions for detecting a confounder when they use different categorizations. Such a factor should be looked at more carefully. We should further consider confounders from the general principle as in Miettinen and Cook (1981): the criterion for detecting a confounder should depend on neither the measure of association chosen nor the categorization taken.

\subsection{Definitions}

Let $\omega$ be a non-empty subset of values of $C$, i.e. $\omega \subseteq \Delta$ and $\omega \neq \varnothing$, where $\Delta$ is the finest original categorization of $C$. A coarse subpopulation of $\omega$ consists of all subpopulations of $C=k$ for all $k \in \omega$. Confounding bias in a coarse subpopulation of $\omega$ is defined as

$$
B_{\omega}=P\left(D_{\bar{e}}=1 \mid E=e, C \in \omega\right)-P\left(D_{\bar{e}}=1 \mid E=\bar{e}, C \in \omega\right) .
$$

Let $p=\left\{\omega_{1}, \ldots, \omega_{s}\right\}$ for $s \geqslant 2$ be a partition of $\Delta=\{1, \ldots, K\}$, a class of non-empty subsets of $\Delta$ such that 
(a) $\omega_{i} \neq \varnothing$ for all $i$,

(b) $\omega_{i} \cap \omega_{j}=\varnothing$ for all $i \neq j$ and

(c) $\cup_{i} \omega_{i}=\Delta$.

The standardized $P_{p}\left(D_{\bar{e}}=1 \mid E=\bar{e}\right)$ based on $p$ is

$$
P_{p}\left(D_{\bar{e}}=1 \mid E=\bar{e}\right)=\sum_{\omega \in p} P\left(D_{\bar{e}}=1 \mid E=\bar{e}, C \in \omega\right) P(C \in \omega \mid E=e) .
$$

Definition 3. A covariate $C$ is called an occasional confounder if there is a partition $p$ such that

$$
\left|P\left(D_{\bar{e}}=1 \mid E=e\right)-P_{p}\left(D_{\bar{e}}=1 \mid E=\bar{e}\right)\right|<|B| .
$$

If $C$ is an occasional confounder, there is a partition such that confounding can be reduced or eliminated by controlling for $C$ with respect to the partition $p$, but such a partition cannot be recognized from the observed data. If $C$ is not an occasional confounder, then confounding cannot be reduced by controlling for $C$, no matter what categorization is chosen for $C$, or no matter how the subpopulations are grouped. Thus it is unnecessary to consider how to control for a factor which is not an occasional confounder. If $C$ is an occasional confounder, then any set containing $C$ is also an occasional confounder. Any confounder is also an occasional confounder, but the reverse is not true.

Definition 4. A factor $C$ is a uniformly irrelevant factor if, for any possible partition $p$,

$$
P_{p}\left(D_{\bar{e}}=1 \mid E=\bar{e}\right)=P\left(D_{\bar{e}}=1 \mid E=\bar{e}\right) .
$$

Hence adjusting for a uniformly irrelevant factor $C$ does not reduce or enhance confounding under any categorization. A uniformly irrelevant factor is not an occasional confounder and thus is not a confounder.

\subsection{Criterion}

Researchers clearly take the concept of a uniformly irrelevant factor as the criterion for screening out a confounder, but in practice they detect a confounder only under a special categorization of a factor. The original idea of Miettinen and Cook's (1981) criterion does not restrict a confounder to a particular categorization, and the criterion essentially aims at the occasional confounder.

Theorem 1. $C$ is a uniformly irrelevant factor if and only if

(a) $C \Perp E$ or

(b) $D_{\bar{e}} \Perp C \mid E=\bar{e}$.

Proof. For the proof of theorem 1, see Appendix A.

If a factor $C$ satisfies (a) or (b) in theorem 1, controlling for it does not reduce confounding no matter how $C$ is categorized, and thus we have the following result.

Corollary 1. If $C$ is an occasional confounder, then

(a) $C \not \Perp E$ and

(b) $D_{\bar{e}} \not \perp C \mid E=\bar{e}$.

This analytically proves the criterion of Miettinen and Cook (1981) obtained inductively. Consider again the example given in Section 3.1. The risk differences in Table 1 are collapsible over groups of 10 years, while $C$ satisfies Miettinen and Cook's criterion. It can be seen from the example that $C$ is an occasional confounder. 


\section{Multiple potential confounders}

In this section, we consider the extension of Miettinen and Cook's (1981) criterion to situations with multiple potential confounders. Fisher and Patil (1974) and Greenland and Robins (1986) realized that, if more than one factor is under consideration, Miettinen and Cook's criterion should be applied conditionally on all the other factors.

Robins (1997) generalized Miettinen and Cook's criterion under the assumption that a known set of potential confounders is sufficient for the control of confounding. Let $C$ be a set of potential confounders, $S$ be a subset of $C$ and $R$ be the remainder subset, i.e. $R=C \backslash S$. Under the assumption that $C$ is sufficient for the control of confounding (i.e. $D_{\bar{e}} \Perp E \mid C$ ), Robins (1997) showed that, if $R$ can be decomposed into disjoint subsets $R_{1}$ and $R_{2}$ such that both

$$
R_{1} \Perp E \mid S \quad \text { and } \quad R_{2} \Perp D_{E} \mid\left(E, R_{1}, S\right)
$$

hold, then $R$ can be deleted from the set of potential confounders, i.e. $R$ is not a confounder set. Greenland, Pearl and Robins (1999) pointed out that Robins's criterion requires both of the above independences simultaneously, but that Miettinen and Cook's criterion only requires one of two analogous independences.

We first give several definitions of confounders and irrelevant factors for situations with multiple potential confounders. Then, without Robins's (1997) assumption of a sufficient covariate set for the control of confounding, we obtain two conditions for deleting $R$ from the set of potential confounders and potential occasional confounders, one of which is just Robins's criterion.

Let $\Delta_{A}$ be the set of all possible values of the covariate set $A$, and define the standardization conditional on $S=s$ by adjusting the distribution of $A$ in the unexposed population to that in the exposed population as

$$
P_{A}\left(D_{\bar{e}}=1 \mid E=\bar{e}, S=s\right)=\sum_{a \in \Delta_{A}} P\left(D_{\bar{e}}=1 \mid E=\bar{e}, A=a, S=s\right) P(A=a \mid E=e, S=s) .
$$

Similarly to the discussion in Sections 2 and 3, we give definitions of an irrelevant factor and a confounder for situations with multiple potential confounders. We say that $R$ is a confounder set conditional on $S=s$ if

$$
\left|P\left(D_{\bar{e}}=1 \mid E=e, S=s\right)-P_{R}\left(D_{\bar{e}}=1 \mid E=\bar{e}, S=s\right)\right|<\left|B_{S}\right|,
$$

where $B_{\mathrm{s}}$ is the confounding bias in the subpopulation $S=s$.

Definition 5. $R$ is an irrelevant set conditional on $S=s$ if

$$
P_{R}\left(D_{\bar{e}}=1 \mid E=\bar{e}, S=s\right)=P\left(D_{\bar{e}}=1 \mid E=\bar{e}, S=s\right) .
$$

An irrelevant set $R$ is not a confounder set. An adjustment for $R, P_{R}\left(D_{\bar{e}}=1 \mid E=\bar{e}, S=s\right)$, cannot reduce confounding bias in the subpopulation $S=s$.

We say that $R$ contains at least one occasional confounder conditional on $S=s$ if there are both a subset $F=\left\{F_{1}, \ldots, F_{m}\right\}$ of $R$ (i.e. $F \subseteq R$ ) and a partition $p_{k}=\left\{\omega_{k 1}, \ldots, \omega_{k I_{k}}\right\}$, $k=1, \ldots, m$, of $\Delta_{F_{k}}$ for each covariate $F_{k}$ in $F$ such that

$$
\left|P\left(D_{\bar{e}}=1 \mid E=e, S=s\right)-P_{F p}\left(D_{\bar{e}}=1 \mid E=\bar{e}, S=s\right)\right|<\left|B_{s}\right|,
$$

where 


$$
\begin{aligned}
P_{F_{p}}\left(D_{\bar{e}}=1 \mid E=\bar{e}, S=s\right)= & \sum_{\omega_{1} \in p_{1}} \ldots \sum_{\omega_{m} \in p_{m}} P\left(D_{\bar{e}}=1 \mid E=\bar{e}, F_{1} \in \omega_{1}, \ldots, F_{m} \in \omega_{m}, S=s\right) \\
& \times P\left(F_{1} \in \omega_{1}, \ldots, F_{m} \in \omega_{m} \mid E=e, S=s\right) .
\end{aligned}
$$

Definition 6. $R$ is a uniformly irrelevant set conditional on $S=s$ if

$$
P_{F p}\left(D_{\bar{e}}=1 \mid E=\bar{e}, S=s\right)=P\left(D_{\bar{e}}=1 \mid E=\bar{e}, S=s\right)
$$

for any $F \subseteq R$ and any partition $p_{k}$ of each $F_{k}$ in $F$.

Definitions 5 and 6 imply that a uniformly irrelevant set must be an irrelevant set and that a uniformly irrelevant set does not contain any occasional confounders.

Theorem 2. If $R$ can be decomposed into two disjoint subsets $R_{1}$ and $R_{2}$ such that either

(a) $R_{1} \Perp E \mid S=s$ and $R_{2} \Perp D_{\bar{e}} \mid\left(E=\bar{e}, R_{1}, S=s\right)$

or

(b) $R_{2} \Perp E \mid\left(R_{1}, S=s\right)$ and $R_{1} \Perp D_{\bar{e}} \mid(E=\bar{e}, S=s)$,

then $R$ is an irrelevant set conditional on $S=s$ and $R_{1}$ is a uniformly irrelevant set conditional on $S=s$.

Proof. For a proof of theorem 2 see Appendix B.

Both conditions (a) and (b) of theorem 2 are testable from data. If the set $R$ satisfies either of them, then we can delete $R$ from the set of potential confounders and delete $R_{1}$ from the set of occasional confounders. Condition (a) is the same as Robins's (1997) criterion given above. We further show that neither adjustment for a subset of $R_{1}$ nor recategorization of some covariates in $R_{1}$ can reduce confounding bias.

The following result gives the necessary conditions for $R$ to contain an occasional confounder.

Corollary 2. If $R$ contains at least one occasional confounder conditional on $S=s$, then

$$
R \not \Perp E \mid S=s \quad \text { and } R \not 1 D_{\bar{e}} \mid(E=\bar{e}, S=s) .
$$

Proof. Suppose that $R \Perp E \mid S=s$ or $R \Perp D_{\bar{e}} \mid(E=\bar{e}, S=s)$. From theorem 2 with $R_{2}=\varnothing$, we know that $R=R_{1}$ is a uniformly irrelevant set and thus does not contain any occasional confounders conditional on $S=s$.

From theorem 2, we can also obtain the following necessary conditions for a confounder set.

Corollary 3. If $R$ is a confounder set conditional on $S=s$, then both

(a) $R_{1} \not \perp E \mid S=s$ or $R_{2} \not \perp D_{\bar{e}} \mid\left(E=\bar{e}, R_{1}, S=s\right)$

and

(b) $R_{2} \not \Perp E \mid\left(R_{1}, S=s\right)$ or $R_{1} \not \Perp D_{\bar{e}} \mid(E=\bar{e}, S=s)$,

for any possible decomposition $R_{1}$ and $R_{2}$ of $R$.

\section{Concluding remarks}

The criteria discussed in this paper are mainly concerned with the comparability-based criterion of Miettinen and Cook (1981), but the definitions are general and can be used 
for both comparability-based and collapsibility-based approaches. Without the untestable assumptions that are necessary for causal inference, confounders cannot be definitely determined, but we can eliminate factors which are not confounders from the set of potential confounders. Miettinen and Cook's criterion can be used to identify uniformly irrelevant factors, whereas the collapsibility-based approach only identifies irrelevant factors, which may also be occasional confounders. The collapsibility-based approach is also commonly employed in epidemiological studies. Miettinen and Cook (1981) and Greenland (1996) showed by counter-example that non-confounding is neither necessary nor sufficient for collapsibility of odds ratios or rate ratios. We are working towards a comparison of the comparability- and the collapsibility-based criteria based on risk ratios and risk differences.

\section{Acknowledgements}

The authors would like to thank Jinling Tang, MD, for helpful discussions and Maria Kocherginsky for helpful comments on a draft of the paper. We also would like to thank the Joint Editor and referees for comments that greatly improved the presentation of the paper. This research was supported by the National Natural Science Foundation of China, National Science Foundation of China for Excellent Youths, a seed grant from the Committee of Research and Conference Grants from the University of Hong Kong and the Trans-Century Training Programme Foundation for the Talents by the State Education Commission.

\section{Appendix A: Proof of theorem 1}

To prove theorem 1, we first show two lemmas.

Lemma 1. For any partition $p=\left\{\omega_{1}, \ldots, \omega_{s}\right\}$ of $\Delta$

$$
B=\sum_{i=1}^{s} B_{\omega_{i}} P\left(C \in \omega_{i} \mid E=e\right)+\alpha_{p}
$$

where

$$
\alpha_{p}=\sum_{i=1}^{s} P\left(D_{\bar{e}}=1 \mid E=\bar{e}, C \in \omega_{i}\right)\left\{P\left(C \in \omega_{i} \mid E=e\right)-P\left(C \in \omega_{i} \mid E=\bar{e}\right)\right\} .
$$

The proof of this lemma follows from simple manipulation.

Lemma 2. $\alpha_{p}=0$ for any partition $p$ of $\Delta$ if and only if $C \Perp E$ or $D_{\bar{e}} \Perp C \mid E=\bar{e}$.

Proof. For the sufficiency, it is immediately obtained that $\alpha_{p}=0$ if $C \Perp E$ or $D_{\bar{e}} \Perp C \mid E=\bar{e}$.

For the necessity, let $p_{k}=\{\{k\}, \Delta \backslash\{k\}\}$ for any $k \in \Delta$. Rewrite $\alpha_{p_{k}}$ as

$$
\begin{aligned}
& P\left(D_{\bar{e}}=1 \mid E=\bar{e}, C=k\right)\{P(C=k \mid E=e)-P(C=k \mid E=\bar{e})\} \\
& \quad+P\left(D_{\bar{e}}=1 \mid E=\bar{e}, C \in \Delta \backslash\{k\}\right)\{P(C \in \Delta \backslash\{k\} \mid E=e)-P(C \in \Delta \backslash\{k\} \mid E=\bar{e})\} \\
& \quad=\left\{P\left(D_{\bar{e}}=1 \mid E=\bar{e}, C=k\right)-P\left(D_{\bar{e}}=1 \mid E=\bar{e}, C \in \Delta \backslash\{k\}\right)\right\}\{P(C=k \mid E=e)-P(C=k \mid E=\bar{e})\} .
\end{aligned}
$$

For $\alpha_{p_{k}}=0$, we obtain

(a) $P\left(D_{\bar{e}}=1 \mid E=\bar{e}, C \in \Delta \backslash\{k\}\right)=P\left(D_{\bar{e}}=1 \mid E=\bar{e}, C=k\right)$ or

(b) $P(C=k \mid E=\bar{e})=P(C=k \mid E=e)$.

We rewrite result (a) as

$$
\frac{P\left(D_{\bar{e}}=1, C \in \Delta \backslash\{k\} \mid E=\bar{e}\right)}{P(C \in \Delta \backslash\{k\} \mid E=\bar{e})}=\frac{P\left(D_{\bar{e}}=1, C=k \mid E=\bar{e}\right)}{P(C=k \mid E=\bar{e})} .
$$

By denoting it as $a / b=c / d$, this equation is equivalent to $(a+c) /(b+d)=c / d$, i.e. 


$$
P\left(D_{\bar{e}}=1 \mid E=\bar{e}\right)=\frac{P\left(D_{\bar{e}}=1, C=k \mid E=\bar{e}\right)}{P(C=k \mid E=\bar{e})} .
$$

Thus we obtain that result (a) is equivalent to

$$
P\left(D_{\bar{e}}=1 \mid E=\bar{e}\right)=P\left(D_{\bar{e}}=1 \mid E=\bar{e}, C=k\right) .
$$

Let

$$
\begin{gathered}
x=P\left(D_{\bar{e}}=1 \mid E=\bar{e}\right), \\
x_{k}=P\left(D_{\bar{e}}=1 \mid E=\bar{e}, C=k\right), \\
y_{k}=P(C=k \mid E=e)-P(C=k \mid E=\bar{e}) .
\end{gathered}
$$

Then the equivalent result to (a) or (b) can be rewritten as

(i) $x=x_{k}$ or

(ii) $y_{k}=0$

for all $k \in \Delta$.

If $C \not \Lambda E$, then there exists $k_{0} \in \Delta$, such that $P\left(C=k_{0} \mid E=\bar{e}\right) \neq P\left(C=k_{0} \mid E=e\right.$ ) (i.e. $y_{k_{0}} \neq 0$ ).

Without loss of generality, assume that $y_{1} \neq 0$; then $x_{1}=x$ by results (i) and (ii). We prove below that $x_{k}=x$ for all $k$.

When $K=2, y_{1} \neq 0$ implies that $y_{2} \neq 0$ for $y_{1}=-y_{2}$, and thus we obtain from results (i) and (ii) that $x_{1}=x_{2}=x$. When $K>2$, assume that $x_{k} \neq x$ for some $k$. Then $y_{k}=0$ by results (i) and (ii). Let $p_{k}^{\prime}=\{\{1, k\}, \Delta \backslash\{1, k\}\}$. Similarly to $\alpha_{p_{k}}$, we can rewrite $\alpha_{p_{k}^{\prime}}$ as

$$
\begin{aligned}
\left\{P \left(D_{\bar{e}}=\right.\right. & \left.1 \mid E=\bar{e}, C \in\{1, k\})-P\left(D_{\bar{e}}=1 \mid E=\bar{e}, C \in \Delta \backslash\{1, k\}\right)\right\} \\
& \times\{P(C \in\{1, k\} \mid E=e)-P(C \in\{1, k\} \mid E=\bar{e})\} .
\end{aligned}
$$

Since $\alpha_{p_{t}^{\prime}}=0$ and $P(C \in\{1, k\} \mid E=e)-P(C \in\{1, k\} \mid E=\bar{e})=y_{1}+y_{k}=y_{1} \neq 0$, we obtain that $P\left(D_{\bar{e}}=1 \mid E=\bar{e}, C \in \Delta \backslash\{1, k\}\right)=P\left(D_{\bar{e}}=1 \mid E=\bar{e}, C \in\{1, k\}\right)$. We have

$$
\begin{aligned}
P\left(D_{\bar{e}}=1 \mid E=\bar{e}, C \in \Delta \backslash\{1, k\}\right)= & \frac{\sum_{i \in \Delta \backslash\{1, k\}} P\left(D_{\bar{e}}=1 \mid E=\bar{e}, C=i\right) P(C=i \mid E=\bar{e})}{\sum_{i \in \Delta \backslash\{1, k\}} P(C=i \mid E=\bar{e})} \\
= & \frac{x-\sum_{i \in\{1, k\}} P\left(D_{\bar{e}}=1 \mid E=\bar{e}, C=i\right) P(C=i \mid E=\bar{e})}{1-\sum_{i \in\{1, k\}} P(C=i \mid E=\bar{e})} \\
= & \frac{x-x_{1} z_{1}-x_{k} z_{k}}{1-z_{1}-z_{k}} .
\end{aligned}
$$

where $z_{k}=P(C=k \mid E=\bar{e})$. Also

$$
\begin{aligned}
& P\left(D_{\bar{e}}=1 \mid E=\bar{e}, C \in\{1, k\}\right)= \frac{\sum_{i \in\{1, k\}} P\left(D_{\bar{e}}=1 \mid E=\bar{e}, C=i\right) P(C=i \mid E=\bar{e})}{\sum_{i \in\{1, k\}} P(C=i \mid E=\bar{e})} \\
&=\frac{x_{1} z_{1}+x_{k} z_{k}}{z_{1}+z_{k}} .
\end{aligned}
$$

Thus we obtain

$$
\frac{x-x_{1} z_{1}-x_{k} z_{k}}{1-z_{1}-z_{k}}=\frac{x_{1} z_{1}+x_{k} z_{k}}{z_{1}+z_{k}} .
$$


For $x_{1}=x$, we can show from this equation that $x_{k}=x$, which contradicts the assumption of $x_{k} \neq x$.

Thus we have proved that $x_{k}=x$ for all $k$, i.e. $D_{\bar{e}} \Perp C \mid E=\bar{e}$.

\section{A.1. Proof of theorem 1}

Define $B_{p}=P\left(D_{\bar{e}}=1 \mid E=e\right)-P_{p}\left(D_{\bar{e}}=1 \mid E=\bar{e}\right)$. Then we have

$$
B_{p}=\sum_{i=1}^{s} B_{\omega_{i}} P\left(C \in \omega_{i} \mid E=e\right) .
$$

By lemma 1, we obtain $B-B_{p}=\alpha_{p}$. From the definition of a uniformly irrelevant factor, we have that $C$ is a uniformly irrelevant factor if and only if $\alpha_{p}=0$ for any $p$. From lemma 2, it is equivalent to

(a) $C \Perp E$ or

(b) $D_{\bar{e}} \Perp C \mid E=\bar{e}$.

\section{Appendix B: Proof of theorem 2}

We first show that $R$ is an irrelevant set conditional on $S=s$. By the definition of standardization, we have

$$
\begin{aligned}
P_{R}\left(D_{\bar{e}}=1 \mid E=\bar{e}, S=s\right)= & \sum_{r_{1} \in \Delta_{R_{1}} r_{2} \in \Delta_{R_{2}}} P\left(D_{\bar{e}}=1 \mid E=\bar{e}, R_{1}=r_{1}, R_{2}=r_{2}, S=s\right) \\
& \times P\left(R_{2}=r_{2} \mid E=e, R_{1}=r_{1}, S=s\right) P\left(R_{1}=r_{1} \mid E=e, S=s\right) .
\end{aligned}
$$

For condition (a), first applying the second conditional independence and then the first, we obtain

$$
\begin{aligned}
P_{R}\left(D_{\bar{e}}=1 \mid E=\bar{e}, S=s\right)= & \sum_{r_{1} \in \Delta_{R_{1}}} P\left(D_{\bar{e}}=1 \mid E=\bar{e}, R_{1}=r_{1}, S=s\right) \\
& \times P\left(R_{1}=r_{1} \mid E=e, S=s\right)=P\left(D_{\bar{e}}=1 \mid E=\bar{e}, S=s\right) .
\end{aligned}
$$

Similarly, the sufficiency of condition (b) can be shown by first applying the first conditional independence and then applying the second.

Next we show that $R_{1}$ is a uniformly irrelevant set conditional on $S=s$. For condition (a), we have that $R_{1} \Perp E \mid S=s$ implies $F \Perp E \mid S=s$ for any $F \subseteq R_{1}$. Thus we obtain

$$
\begin{aligned}
P_{F_{p}}\left(D_{\bar{e}}=1 \mid E=\bar{e}, S=s\right)= & \sum_{\omega_{1} \in p_{1}} \ldots \sum_{\omega_{m} \in p_{m}} P\left(D_{\bar{e}}=1 \mid E=\bar{e}, F_{1} \in \omega_{1}, \ldots, F_{m} \in \omega_{m}, S=s\right) \\
& \times P\left(F_{1} \in \omega_{1}, \ldots, F_{m} \in \omega_{m} \mid E=\bar{e}, S=s\right)=P\left(D_{\bar{e}}=1 \mid E=\bar{e}, S=s\right) .
\end{aligned}
$$

Similarly, the sufficiency of condition (b) can be obtained since $R_{1} \Perp D_{\bar{e}} \mid(E=\bar{e}, S=s)$ implies $F \Perp D_{\bar{e}} \mid(E=\bar{e}, S=s)$ for any $F \subseteq R_{1}$.

\section{References}

Boivin, J. F. and Wacholder, S. (1985) Conditions for confounding of the risk ratio and of the odds ratio. Am. J. Epidem., 121, 152-158.

Breslow, N. E. and Day, N. E. (1980) Statistical Methods in Cancer Research, vol. I, The Analysis of Case-Control Studies. Lyon: International Agency for Research on Cancer.

Dawid, A. P. (1979) Conditional independence in statistical theory (with discussion). J. R. Statist. Soc. B, 41, 1-31.

Ducharme, G. R. and LePage, Y. (1986) Testing collapsibility in contingency tables. J. R. Statist. Soc. B, 48, 197-205.

Fisher, L. and Patil, K. (1974) Matching and unrelatedness. Am. J. Epidem., 100, 347-349.

Geng, Z. (1992) Collapsibility of relative risk in contingency tables with a response variable. J. R. Statist. Soc. B, 54, 585-593.

Grayson, D. A. (1987) Confounding confounding. Am. J. Epidem., 126, 546-553.

Greenland, S. (1989) Reader reactions: confounding in epidemiologic studies. Biometrics, 45, 1309-1310.

(1996) Absence of confounding does not correspond to collapsibility of the rate ratio or rate difference. Epidemiology, 7, 498-501.

Greenland, S., Morgenstern, H., Poole, C. and Robins, J. M. (1989) Re: "Confounding confounding”. Am. J. Epidem., 129, 1086-1091. 
Greenland, S., Pearl, J. and Robins, J. M. (1999) Causal diagrams for epidemiologic research. Epidemiology, 10, $37-48$.

Greenland, S. and Robins, J. M. (1986) Identifiability, exchangeability, and epidemiological confounding. Int. J. Epidem., 15, 413-419.

Greenland, S., Robins, J. M. and Pearl, J. (1999) Confounding and collapsibility in causal inference. Statist. Sci., 14, 29-46.

Guo, J. and Geng, Z. (1995) Collapsibility of logistic regression coefficients. J. R. Statist. Soc. B, 57, $263-267$.

Holland, P. W. (1986) Statistics and causal inference (with discussion). J. Am. Statist. Ass., 81, 945-970. (1989) Reader reactions: confounding in epidemiologic studies. Biometrics, 45, 1310-1316.

Kleinbaum, D. G., Kupper, L. L. and Morgenstern, H. (1982) Epidemiologic Research: Principles and Quantitative Methods. New York: Van Nostrand Reinhold.

Miettinen, O. S. (1972) Standardization of risk ratios. Am. J. Epidem., 96, 383-388.

Miettinen, O. S. and Cook, E. F. (1981) Confounding: essence and detection. Am. J. Epidem., 114, $593-603$.

Pearl, J. (1995) Causal diagrams for empirical research (with discussion). Biometrika, 83, 669-710.

Robins, J. M. (1989) The control of confounding by intermediate variables. Statist. Med., 8, 679-701. (1997) Causal inference from complex longitudinal data. In Latent Variable Modeling with Applications to Causality (ed. M. Berkane), pp. 69-117. New York: Springer.

Robins, J. M. and Morgenstern, H. (1987) The foundations of confounding in epidemiology. Comput. Math. Applic., 14, 869-916.

Rosenbaum, P. R. and Rubin, D. B. (1983) The central role of the propensity score in observational studies for causal effects. Biometrika, 70, 41-55.

Rothman, K. J. (1986) Modern Epidemiology. Boston: Little, Brown.

Rubin, D. B. (1974) Estimating causal effects of treatments in randomized and non-randomized studies. J. Educ. Psychol., 66, 688-701.

Spirtes, P., Glymour, C. and Scheines, R. (1993) Causation, Prediction and Search. New York: Springer.

Weinberg, C. R. (1993) Toward a clearer definition of confounding. Am. J. Epidem., 137, 1-8.

Whittemore, A. S. (1978) Collapsibility of multidimensional contingency tables. J. R. Statist. Soc. B, 40, 328-340.

Wickramaratne, P. J. and Holford, T. R. (1987) Confounding in epidemiologic studies: the adequacy of the control groups as a measure of confounding. Biometrics, 43, 751-765. 